\title{
Transcriptome profiling of ulcerative colitis mouse model suggests biomarkers and therapeutic targets for human colitis
}

\author{
Yarani, Reza ; Palasca, Oana; Doncheva, Nadezhda Tsankova; Anthon, Christian; Pilecki, Bartosz; \\ Svane, Cecilie A. S. ; Mirza, Aashiq Hussain; Litman, Thomas; Holmskov, Uffe; Bang-Berthelsen, Claus
} Heiner

Total number of authors:

14

Published in:

bioRxiv

Link to article, DOI:

10.1101/2020.08.12.225458

Publication date:

2020

Document Version

Publisher's PDF, also known as Version of record

Link back to DTU Orbit

Citation (APA):

Yarani, R., Palasca, O., Doncheva, N. T., Anthon, C., Pilecki, B., Svane, C. A. S., Mirza, A. H., Litman, T., Holmskov, U., Bang-Berthelsen, C. H., Vilien, M., Jensen, L. J., Gorodkin, J., \& Pociot, F. (2020). Transcriptome profiling of ulcerative colitis mouse model suggests biomarkers and therapeutic targets for human colitis. bioRxiv. https://doi.org/10.1101/2020.08.12.225458

\section{General rights}

Copyright and moral rights for the publications made accessible in the public portal are retained by the authors and/or other copyright owners and it is a condition of accessing publications that users recognise and abide by the legal requirements associated with these rights.

- Users may download and print one copy of any publication from the public portal for the purpose of private study or research.

- You may not further distribute the material or use it for any profit-making activity or commercial gain

- You may freely distribute the URL identifying the publication in the public portal 


\title{
Transcriptome profiling of ulcerative colitis mouse model suggests biomarkers and therapeutic targets for human colitis
}

\author{
Reza Yarani ${ }^{1^{*}}$, Oana Palasca ${ }^{2,3,4}$, Nadezhda T. Doncheva ${ }^{2,3,4}$, Christian Anthon ${ }^{3,4}$, Bartosz Pilecki ${ }^{5}$, Cecilie A. S. Svane ${ }^{1}$, \\ Aashiq H. Mirza ${ }^{3,6}$, Thomas Litman, Uffe Holmskov ${ }^{5}$, Claus Heiner Bang-Berthelsen ${ }^{8,9}$, Mogens Vilien ${ }^{10}$, Lars J. \\ Jensen $^{2,3}$, Jan Gorodkin ${ }^{3,4}$, Flemming Pociot ${ }^{1,3,11,12^{*}}$
}

\footnotetext{
${ }^{1}$ Translational Type 1 Diabetes Research, Department of Clinical Research, Steno Diabetes Center Copenhagen, Gentofte, Denmark

${ }^{2}$ Novo Nordisk Foundation Center for Protein Research, University of Copenhagen, Copenhagen, Denmark

${ }^{3}$ Center for non-coding RNA in Technology and Health, University of Copenhagen, Copenhagen, Denmark

${ }^{4}$ Department of Veterinary and Animal Sciences, University of Copenhagen, Copenhagen, Denmark

${ }^{5}$ Department of Cancer and Inflammation Research, Institute of Molecular Medicine, Faculty of Health Sciences, University of Southern Denmark, Odense, Denmark

${ }^{6}$ Department of Pharmacology, Weill Cornell Medicine, Cornell University, New York, NY 10065, USA

7 Department of Immunology and Microbiology, University of Copenhagen, Copenhagen, Denmark

${ }^{8}$ Research Group for Microbial Biotechnology and Biorefining, National Food Institute, Technical University of Denmark, Kgs. Lyngby, Denmark

${ }^{9}$ Department of Gastroenterology, North Zealand Hillerød Hospital, Hillerød, Denmark

${ }^{10}$ Department of Surgery, North Zealand Hospital, Hillerød 3400, Denmark

${ }^{11}$ Copenhagen Diabetes Research Center, Department of Pediatrics, Herlev University Hospital, Herlev, Denmark

${ }^{12}$ Department of Clinical Medicine, Faculty of Health and Medical Sciences, University of Copenhagen, Copenhagen, Denmark
}

*All Correspondence to:

Reza Yarani: reza.yarani.01@regionh.dk

Flemming Pociot: flemming.pociot@regionh.dk

\section{Abstract}

BACKGROUND \& AIMS: Ulcerative colitis (UC) is an inflammatory bowel disorder with unknown etiology. Given its complex nature, in vivo studies to investigate its pathophysiology is vital. Animal models play an important role in molecular profiling necessary to pinpoint mechanisms that contribute to human disease. Thus, we aim to identify common conserved gene expression signatures and differentially regulated pathways between human UC and a mouse model hereof, which can be used to identify UC patients from healthy individuals and to suggest novel treatment targets and biomarker candidates.

METHODS: Therefore, we performed high-throughput total and small RNA sequencing to comprehensively characterize the transcriptome landscape of the most widely used UC mouse model, the dextran sodium sulfate (DSS) model. We used this data in conjunction with publicly available human UC transcriptome data to compare gene expression profiles and pathways.

RESULTS: We identified differentially regulated protein-coding genes, long non-coding RNAs and microRNAs from colon and blood of UC mice and further characterized the involved pathways and biological processes through which these genes may contribute to disease development and progression. By integrating human and mouse UC datasets, we suggest a set of 51 differentially regulated genes in UC colon and blood that may improve molecular phenotyping, aid in treatment decisions, drug discovery and the design of clinical trials.

CONCLUSION: Global transcriptome analysis of the DSS-UC mouse model supports its use as an efficient highthroughput tool to discover new targets for therapeutic and diagnostic applications in human UC through identifying relationships between gene expression and disease phenotype.

KEYWORDS: DSS; UC; Transcriptomics; Colon; Blood 


\section{Introduction}

Ulcerative colitis (UC) is an inflammatory bowel disorder that mainly affects the large intestine. Most often, UC initiates from the rectum and affects the mucosal lining and is limited to the innermost layers of the large intestine. Multiple pathogenic factors, including numerous susceptibility gene variants, environmental factors, changes in the gut microbiota and a dysregulated immune response are all associated with UC. Despite this recognition and the identification of apparently relevant factors, a complete understanding of UC pathogenesis is still out of reach and, thus, treatment may not be optimal. An important reason for this unsatisfactory situation is the currently limited comprehension of what the truly relevant components of UC immunopathogenesis are. Thus, given the complex nature of UC, the study of animal models is crucial. A big challenge in using animal models for UC is whether the model's genes and molecular pathways are analogous to those in humans. Although many coding and non-coding genes between humans and mice are highly similar, ${ }^{1}$ they might be regulated differently, and also the pathophysiology may be different. Expression of both differentially regulated coding and non-coding genes contributes to disease pathology. ${ }^{2,3}$ Differentially regulated long non-coding RNAs (IncRNAs) and microRNAs (miRNAs) are linked to the change in the expression of many protein coding genes (PCGs) and subsequently, development of disease. Many studies are made without taking the non-coding RNAs, i.e. disease related pathways and differentially expressed genes, into account and thereby, fundamental and essential players in the disease regulatory networks may be overlooked. ${ }^{4}$

Preclinical murine models have been used for in vivo assessments of UC, and one of the best established UC models is the dextran sulfate sodium (DSS) model. ${ }^{5,6}$ DSS is a negatively charged polysaccharide with colitogenic properties that induces colitis when given orally. ${ }^{5}$ Although the mechanisms by which DSS induces intestinal inflammation are not fully understood, ${ }^{7}$ it seems that the exposed colonic monolayer of epithelial cells in the large intestine, specifically the distal colon are being progressively eroded and the lamina propria barrier integrity compromised, in which an enormous number of microorganisms live. Therefore, inflammation is triggered following bacteria (and their products) translocation across the damaged intestinal wall. To our knowledge, a comprehensive investigation of the coding and non-coding colonic and blood transcriptome of the DSS model and its comparison with human UC has not been performed.

We therefore set out to characterize the transcriptomic landscape of the DSS-UC mouse model by performing transcriptomic profiling of PCGs, IncRNAs and miRNAs from colon and whole blood using deep RNA sequencing. We identified differentially expressed IncRNAs and underscored their functional importance by highlighting their functional annotation through the expressed neighboring PCGs. Then we identified the targets for the differentially regulated miRNAs and identified biological processes that these targets are affecting. Further, we looked at the underlying molecular pathways and networks and assessed the similarity between the experimental model and human UC. Our results identified widespread differential regulation of coding and non-coding genes both in the mouse model and human UC with marked similarities in both tissues specifically in colon. Altogether, this study offers a great opportunity to further investigate the role of novel and previously identified differentially regulated genes in UC and to improve molecular phenotyping, which may have marked effects on treatment decisions, drug discovery and the design of clinical trials. 


\section{Materials \& Methods}

\subsection{Mouse model, RNA sequencing, data processing and differential expression analysis}

Ulcerative colitis was induced using dextran sulfate sodium salt (DSS) in C57BL/6 male mice. The control group was time-matched and received the same drinking water without DSS. Disease activity index (DAI) was recorded. RNA was extracted from colon and whole blood, and total and small RNA were sequenced using the Illumina HiSeq 4000 system. Gene-level quantification corresponding to the total RNA-Seq data was obtained using Stringtie, ${ }^{8}$ and mature miRNA expression was quantified using miRDeep2. ${ }^{9}$ Differential expression was performed using DESeq2. ${ }^{10}$ (Supplementary Methods). The raw total and small RNA-Seq data have been deposited in the Gene Expression Omnibus (GEO) database with accession number GSE155303.

\subsection{Functional enrichment and network analysis of differentially expressed genes}

Functional enrichment analysis on the significantly differentially expressed (SDE) genes (FC > 2, padj $\leq 0.05$ ) was performed using stringApp ${ }^{11}$ in Cytoscape ${ }^{12}$ as well as Ingenuity pathway analysis (IPA) software. ${ }^{13}$

To suggest a functional role for SDE IncRNAs, we performed enrichment on their genomic neighbor genes situated within a span of $100 \mathrm{~kb}$ upstream and downstream of the IncRNA. For SDE miRNAs (padj $\leq 0.05$ ), we retrieved a set of miRNA-target genes and performed enrichment on them (Supplementary Methods).

stringApp was used to retrieve STRING networks ${ }^{14}$ in Cytoscape for the set of SDE PCGs in mouse colon and blood and for the SDE PCGs conserved in humans. In order to rank the SDE genes common between mouse and human colon and blood based on their importance, weighted centrality analysis was performed on their STRING network (Supplementary Methods).

\subsection{Comparison between mouse and human data}

In order to compare our mouse data with data from UC patients, we obtained and processed data from 8 different publicly available studies. ${ }^{15-21} \mathrm{~A}$ set of SDE genes in colon and blood was obtained by combining the different datasets, requiring each gene to be consistently SDE (padj $\leq 0.05$ ) in at least two datasets. For the small RNA-Seq we only used two datasets and required that each miRNA is SDE (padj $\leq 0.05$ ) in at least one of these datasets. The final SDE human lists were intersected with the mouse SDE blood and colon gene sets (padj $\leq 0.05$ ) using orthology relationships extracted from Ensembl (release 97), ${ }^{22}$ and HCOP database ${ }^{23,24}$ for IncRNAs (Supplementary Methods).

\subsection{Validation of SDE genes by quantitative real-time PCR}

The expression of 19 selected SDE genes common in both mouse and human colon and blood was validated by quantitative real-time PCR (qRT-PCR). For validation, colon and blood cDNAs from 5 DSS-UC mice, 5 control mice, 10 UC patients and 6 controls were used. Detailed information on patient demographics and primer sequences used for qRT-PCR can be found in Supplementary File 1 and procedure detail in the supplementary section. Expression of each gene tested was represented as a FC using the $2^{-\triangle \triangle C T}$ method. GUSB, B2M, ACTB and TBP were used as the reference genes for normalization. 


\section{Results}

\subsection{Transcriptional profile of UC mouse model}

Successful colitis induction in mice was confirmed by combined DAI score, measuring inflammatory markers, histological and micro positron emission tomography imaging analysis (Supplementary Figure 1). For the total RNA-Seq, 40-66M paired-end reads were obtained per sample, with 39-58M in colon and 17-33M in blood, uniquely mapping to the mouse genome. For the small RNA-Seq after cleaning, 15-25M reads were obtained, where 14-20M reads mapped to the set of mouse miRNAs annotated in miRBAse v.22. Principal component analysis (Supplementary Figure 2) shows a clear separation of the disease and control groups on the first principal component in total RNA-Seq colon and blood, as well as small RNA-Seq colon. The separation between the groups is strongly supported by the differential gene expression pattern, suggesting that global gene expression differences can be clearly detected between UC and healthy controls (Table 1 and Supplementary File 2).

Table 1: Numbers of SDE genes identified in colon and blood of DSS-UC mice versus control. (For PCGs and IncRNAs $|\log 2 \mathrm{FC}|>1$, padj $\leq 0.05$ and for miRNAs $\mathrm{FC}>1.5$, padj $\leq 0.05)$.

\subsection{UC mouse colon and blood transcriptomics signature}

We identified 3,061 and 623 SDE genes from the total RNA-Seq and 73 and 10 SDE miRNAs from the small RNA-Seq data comparing UC mice colon and blood with control, respectively (Table 1). While SDE PCGs are mainly upregulated in both tissues, IncRNAs in colon are equally up- and downregulated and the miRNAs in blood are mostly downregulated. The RNA expression profile of the SDE genes and miRNAs, obtained by hierarchical clustering, is shown in heatmaps for colon and blood (Figure 1A, C). The SDE genes and miRNAs are highlighted in the volcano plots for colon and blood (Figure 1B, D).

From the SDE genes in colon, we detected 116 genes which have a human ortholog that is situated within $100 \mathrm{~kb}$ distance of the known Informatory bowel disease (IBD)-risk loci, ${ }^{25}, 26$ i.e. 110 PCGs, 2 IncRNAs and 4 other RNAs. In blood, 30 SDE genes, all PCGs, were IBD-risk loci associated (Supplementary File 3). Several of the SDE genes including Reg3b, Abca12, Sptssb, Prss22, Pitx2, Ceacam12, Myot, Defb37, Gml and Ugt2b5 in colon and Gpr27, Gm7457, Usp2, Stfa3, Gm37800, Dync2li1, Gm32486, Gm7206, Gm33326 and Epha2 in blood have not been identified previously in this model. Top 10 up- and downregulated (padj $\leq 0.05$, avg. exp $>100$ ) genes in colon and blood are listed in (Supplementary Table 1).

Figure 1: Heatmaps of SDE PCGs, IncRNAs and miRNAs, based on z-scores of normalized log counts for (A) colon and (B) blood. Volcano plots - $\log _{2} \mathrm{FC}$ ratio on the $\mathrm{x}$ axis versus - $\log 10$ padj on the $y$ axis for colon (C) and blood (D).

To investigate the roles of the SDE PCGs in UC colon and blood, we performed functional enrichment analysis (supplementary File 4). For mouse colon, the top ranked processes and pathways include immune and inflammatory system, response to inflammation and organ/tissue remodeling (Supplementary Figure 3A). In particular, the KEGG disease pathway for IBD is enriched with almost half of the pathway genes being SDE in our data. Although the number of SDE genes in blood is lower than in colon, the top ranked processes and 
pathways enriched for the SDE genes in blood overlap to a great extent with the colon (Supplementary Figure 3B) and are mainly related to the immune system, inflammatory system and connective tissue.

For the SDE IncRNAs, we performed functional enrichment of their genomic neighbors expressed in our samples (Supplementary Methods). We retrieved a total of 888 PCGs in colon and 159 PCGs in blood (Supplementary File 5). Cancer, organismal injury and abnormalities, gastrointestinal disease and immunological disease were overrepresented for both tissues with stronger significance in colon than in blood (Supplementary File 5).

In colon for 59 out of 73 SDE miRNAs, we identified 2,330 target candidates expressed in our samples and for blood, for 8 out of 10 SDE miRNAs we identified 1,641 targets expressed in our samples (Supplementary Figure 4A, B). Functional enrichment of the targets in colon and blood showed overrepresentation of immune related processes, several infectious and immunological diseases as well as JAK-STAT, thyroid hormone and MAPK signaling pathways (Supplementary File 6).

\subsection{Overlap of SDE genes in colon and blood of the UC mouse}

To identify genes commonly differentially regulated in colon and blood of UC mice with importance in the disease phenotype by overlap, 284 genes were retrieved (Supplementary File 7). Venn diagrams illustrating these genes' relationship in colon and blood and the MA plot are shown in Figure 2A, B. From SDE common PCGs, Prok2 (chemoattractant, labeled as outlier), Saa3 (acute phase apolipoproteins) and Gm49368 (predicted gene) were three most upregulated genes in the colon (logFC), while in blood, $G m 49368$ was the highest followed by Prok2 and Saa3. All 5 common IncRNAs showed significant upregulation with Gm11714 most upregulated in blood and Mirt2 most upregulated in colon (and second-most upregulated in the blood). For the miRNAs, mir-223-3p is the only common SDE (padj $\leq 0.05$ ) showing upregulation in both colon and blood with higher expression in blood than in colon, possibly indicating it is an immune-cell enriched miRNA. However, with a less stringent threshold of padj<0.1, we identify three other common miRNAs, downregulated in both colon and blood: mir-194-5p, mir-196b-5p and mir-215-5p. All three are highly abundant in colon and less abundant in blood and have previously been shown to be colon tissue specific in humans according to the miRNA TissueAtlas database. ${ }^{27}$ mir-223-3p showed to be more abundant in blood compare to the others. ${ }^{27}$

Collectively, the common SDE genes define distinct inflammatory, immune system and connective tissue related gene expression signatures. We observe enrichment of genes in processes related to the immune system, specifically regulation of inflammatory response and leukocyte activation, cytokine-cytokine receptor interactions (Figure 2C, D, Supplementary Figure $3 C$ ). Several KEGG pathways are related to infectious diseases (bacterial, viral and parasitic) that alter the immune response of cells. Analysis using IPA confirms these findings (Supplementary File 7).

Figure 2: (A) Venn diagram of the SDE genes overlap between colon and blood; Other RNAs include pseudogenes, TEC, snoRNA, miscRNA, etc. Contra-regulated genes are commonly SDE genes in both tissues, which are regulated in opposite directions (B) MA Plot showing the relationships between $\log _{2} \mathrm{FC}$ in colon and blood for each of the common SDE genes; $y$ axis indicates whether the gene has a higher $F C$ in colon or blood, $x$ axis reflects the cumulative FCs magnitude in colon and blood. Genes labeled as outlier in each organ were removed from the plot. (C) Top 15 enriched KEGG pathways and (D) Top 15 enriched GO biological processes for the common SDE gene between mouse blood and colon. Gene ratio corresponds to the ratio between the number of common SDE and all expressed genes in the pathway. The size of the 
dot indicates the number of SDE genes (gene counts), while the color represents the enrichment significance as given by the FDR adjusted $p$-values.

\subsection{Comparison between SDE genes in UC mouse colon and blood with human UC}

\subsubsection{Colon and blood transcriptomics signature of human UC}

In order to obtain reliable sets of SDE genes in human UC and to compare them with mouse in both colon and blood, we identified integrated sets of SDE genes using several public human datasets. For colon, 2 RNASeq $^{18}$ and 2 microarray studies ${ }^{19,} 20$ and for blood, 2 RNA-Seq studies ${ }^{16,17}$ were used (Table 2, Supplementary File 8). Human miRNA data for colon was obtained by combining one RNA-Seq and one microarray dataset. We have not been able to identify any high-quality miRNA dataset corresponding to whole blood of UC patients.

Table 2: Public human UC datasets used in our study.

The combined sets of human SDE PCGs and IncRNAs, contained 11,000 genes in colon, and $~ 2,000$ genes in blood (padj $\leq 0.05$ in at least 2 datasets and consistently differentially regulated across datasets). We observed approximately equal proportions of up- and downregulated PCGs in colon, and a predominant ( $75 \%$ ) upregulation in blood. In both colon and blood IncRNAs were mainly ( 60\%) downregulated. Furthermore, $\sim 2,500$ genes in colon and $\sim 130$ genes in blood were inconsistently differentially regulated between the datasets and thus not included in the combined SDE gene sets. By combining the two public small RNA-Seq datasets in colon, ${ }^{15,}{ }^{21}$ we obtained 207 SDE miRNAs with padj $\leq 0.05$ in at least one dataset and 37 were SDE in both datasets. No miRNA was found to be inconsistently differentially regulated between the two datasets (Table 3).

Table 3: Numbers of SDE genes in human colon and blood combined datasets. (For all padj $\leq 0.05$ in $\min 2$ datasets).

\subsubsection{Comparison between SDE genes of human and mouse UC}

Using one-to-one and one-to-many orthology assignments, we compared the combined sets of SDE genes in humans with the sets of SDE genes in mouse colon and blood (Table 4). For colon, almost $75 \%$ of the SDE mouse genes with a human ortholog are also found in the human SDE set and for blood, only $30 \%$ of the SDE mouse genes with human orthologs are found in the human blood SDE gene set. The enrichment analysis for these common SDE PCGs in UC colon and blood showed mainly immune, inflammatory and connective tissue processes (Supplementary File 9 and 10). Seven IncRNAs showed significant up- (H19, DNM3OS, 5430425K12RIK, 3110039I08RIK and FENDRR) and down-regulation (HOXA11OS and HOTTIP) in colon of both mouse and humans, while no common IncRNAs were detected in blood. For 5 out of 7 common colon SDE IncRNAs, 33 neighboring genes (100kb upstream and downstream) were retrieved and they were enriched for terms including connective tissue disorders, organismal injury and abnormalities. From the common miRNAs, 12 were downregulated and 9 were upregulated in the same direction in the colon of mouse and human. Functional enrichment analysis for the 1,468 targets (detected in our samples) of 17 out of 21 SDE 
miRNAs in colon showed enrichment for terms including cancer, organismal injury and abnormalities, gastrointestinal disease, and several immune related processes (Supplementary File 9).

Though $25 \%$ of common SDE genes in colon are contra-regulated, 356 genes with upregulation in mice and downregulation in humans showed to be mainly involved in lipid metabolism, ion transport, regulation of localization, trans-synaptic signaling. This could reflect species-specific differences in biological processes' gene regulation. No enrichment was found for the 133 genes which were downregulated in mice and upregulated in humans. Dio3os was the only IncRNA in colon with upregulation in UC mouse and downregulation in humans. mir-10a-5p, mir-10b-3p and mir-10b-5p were colon miRNAs upregulated in mice and downregulated in humans, and highly expressed in both, while mir-130-3p was downregulated in mice and upregulated in humans, though lower expressed in both organisms compared to the other three miRNAs (Supplementary File 9).

Overall, 51 genes were identified as being commonly SDE between the colon and blood of both human and mouse when compared with the healthy controls (Figure $3 \mathrm{~A}$ ). These genes were all upregulated except for $P P 1 R 3 B$ which is upregulated in blood and downregulated in colon. From these genes, only SLC11A1 and STAT3 were IBD risk loci associated. Collectively, these strictly filtered genes showed to be mainly involved in inflammatory, immunological and connective tissue related processes (Figure 3B, Supplementary File 11). Neither of 5 IncRNAs and 1 miRNA, which were commonly SDE between colon and blood of mouse, were present in the human datasets.

Table 4: Numbers of overlapping genes between UC mouse and UC human colon and blood (For all padj $\leq 0.05$ ). $\uparrow$ : Upregulation in both mouse and human, $\downarrow \downarrow$ : Downregulation in both mouse and human, $\uparrow \downarrow$ : Opposite directions between mouse and human.

From the 51 genes common between human and mouse in both colon and blood, 19 were selected for qPCR validation in a separate human UC cohort and the UC mice. Among these selected genes, 8 were genes that were either novel in a UC phenotype setting or not being previously reported as therapeutic target or biomarkers. Furthermore, they were central in the network of protein-protein associations retrieved from STRING v11 (Figure 3C, Supplementary File 11) as measured by a combination of three complementary centrality measures (degree, closeness and betweenness). While degree accounts for the direct interaction partners of each node, closeness and betweenness measure how central and thus important a node is with respect to all other nodes in the network (Supplementary Methods). The expression pattern of all tested genes by qPCR in colon and blood in both mouse and human validation cohorts nicely matched the RNA-seq analysis (Figure 3D and Supplementary Figure 5). The UC phenotype functional conservation and the importance for direct protein interactions with well-known immune-related and inflammatory genes suggests that these genes have high therapeutic-target and diagnostic application potential.

Figure 3: (A) Gene overlap diagram between human combined sets and mouse colon and blood SDE genes with padj $\leq 0.05$. Both the total number of genes in the sets and orthologous genes (in parenthesis) out of total is shown. (B) Top 15 enriched KEGG pathways (top 15 enriched GO biological processes) for the common SDE genes between human, mouse colon and blood. Gene ratio corresponds to the ratio between the number of common SDE genes in mousehuman and all human genes in the pathway. The size of the dot indicates the SDE genes number (gene counts), while the color represents the enrichment significance as given by the FDR adjusted p-values. (C) Differential gene expression visualization on the STRING network of genes common between mouse (inner node circle) and human (outer node 
circle) in blood (left node half) and colon (right node half). log2FC values are shown with a blue-white-red gradient and dark grey color indicates missing values. The size of the nodes corresponds to their network importance as measured by a combination of degree, closeness and betweenness centrality. (D) To validate the expression level of a few selected genes common in the colon and blood of human and mouse UC, qRT-PCR was performed. S100A8 and S100A9 were used as positive inflammatory controls.

\section{Discussion}

Over the last few years, several transcriptional profiling studies have provided evidence regarding differential regulation of PCGs, IncRNAs and miRNAs in UC. ${ }^{18,20,28,29}$ Given the complex nature of UC, an in vivo model approach to study its etiology is of paramount importance. As a surrogate to human UC, the DSS-UC mouse has been extensively used. However, its transcriptional landscape and its (dis-)similarity to human UC have not yet been studied and comprehensively characterized.

Here, we performed colon and blood transcriptional profiling of UC mice for both coding and non-coding RNAs and investigated the affected biological pathways. Our analyses showed that most of the differentially regulated genes are upregulated and involved in immunological and inflammatory responses as well as connective tissue, organ abnormality and injuries. This clearly points to the pathological nature of UC and importance of inflammatory processes in the destruction of epithelium and aggravation of disease. Moreover, alterations and mainly upregulation of genes which suppress inflammation, both in the colon and blood were detected. Genes, which demonstrated significant downregulation, were mainly genes with basic metabolic functions involved in cellular growth and proliferation, lipid, vitamin, amino acid and mineral metabolism. Most of the genes common between colon and blood of UC mice are regulated in the same direction (upregulated) and are related to immune or inflammatory processes. Among these genes, S100a8, S100a9, II1b, Lcn2, C3, Cxcl2, Nos2, SIpi, Socs3, Ifitm6, Csf2rb, TIr2 and Cd44 have previously been detected in UC mouse models in other studies. ${ }^{30-34}$ Several other SDE genes in colon and blood from our study including Lipg, Gm5483, B230303A05Rik, Ambp and Trem3 have not been shown previously in this model. The largest overlap of 1,566 SDE genes was with a recent study by Czarnewski et al, where deep RNA sequencing ${ }^{35}$ was performed to stratify different subtypes of human UC using the common genes in human and mouse UC. Although they used a very similar approach, neither non-coding RNAs nor blood transcriptome were considered in their study.

As the first study investigating the transcriptional landscape of both IncRNAs and miRNAs in the colon and blood of the DSS-UC mice model, we identified widespread differential regulation of non-coding RNAs. Numerous SDE IncRNAs and miRNAs were identified in both tissues, many of which have not been reported previously. Among SDE IncRNAs in the colon, several are well-known, including H19, Meg3, Hottip, Hoxa11os and Mirt2. The latter was also SDE in blood and has been shown to be a negative regulator of inflammation. ${ }^{36}$ $H 19$ upregulation is believed to significantly decrease the expression of the vitamin D receptor and thus have a destructive effect on the intestinal epithelial barrier function by increasing permeability and decreasing the expression of ZO-1 and occludin tight junction proteins. ${ }^{28,37}$ Not much is known regarding the other IncRNAs in UC settings, but recently Meg3 was shown to inhibit the inflammatory response in ankylosing spondylitis characterized by chronic inflammation. ${ }^{38}$ Hottip silencing in rheumatoid arthritis led to reduced inflammation. ${ }^{39}$ In UC mouse blood IncRNAs Neat1 and Panct2 also showed to be SDE in disease. Neat1 is shown to be pro-inflammatory and its inhibition suppresses the inflammatory response in IBD, ${ }^{40}$ whereas little is known about Panct2. Moreover, numerous SDE IncRNAs not reported before in UC mouse including 
top three upregulated 9130221F21Rik, E230034D01Rik, 1200007C13Rik in colon and Gm7457, Gm37800, Gm32486 in blood and many more up- and downregulated new IncRNAs were identified in our study.

Among the SDE miRNAs, IBD-related miRNAs including miR-30, -223, -21, -142 were upregulated and miR192 was downregulated in colon, while miR-223, -16 and -126 were upregulated in blood. miR-223-3p is the only miR upregulated in both colon and blood of the UC mice. It is upregulated in neutrophils and monocytes and acts as a controller of NLRP3 inflammasome activity, which regulates the intestine inflammatory process by affecting IL-1 $\beta$ production. ${ }^{41}$ It has also been shown that miR-223 mediates the cross talk between intestinal barrier and IL-23 pathway by targeting CLDN8, which is a claudin protein that constitutes the backbone of the intestinal barrier. ${ }^{42}$ miR-223 has also been used as a biomarker in IBD. ${ }^{43}$ Thus, evidence clearly suggests its proinflammatory role and highlights its potential as a RNA biomarker that seems to be conserved between different species. It is noteworthy that in the current study, many new SDE miRNAs not shown in this UC mouse model previously including top three upregulated mir-615-3p, mir-212-3p, mir-224$5 p$ in colon and mir-149-5p in blood and many more are identified, which need future detailed functional investigations.

By comparing our UC mouse to human UC data, we identified similar widespread directional differential regulation of genes. The overlap of a substantial number of differentially regulated genes in mouse and human UC suggests fundamental common mechanisms responsible for disease onset and progression. To date, few studies using colon have compared the transcriptional changes in UC mouse models and human UC. ${ }^{33-35}$ Holgersen et al. identified 92 SDE genes in both human CD and UC, ${ }^{33}$ which were used for comparison to their three mouse models. In the DSS model, since colon samples were pooled, no statistical significance of differences could be calculated between disease and healthy mice. However, 59 genes showed to be common between our lists. In their study, non-coding RNAs and blood transcriptome were not studied. Rankin et al., however, identified 12 PCGs and 6 IncRNAs associated with DSS mouse colitis and human UC. ${ }^{34}$ No small RNA and blood profiling were performed in their study. In the Czarnewski et al study, 650 SDE genes common between human and mouse UC colon were identified, but no non-coding RNA and blood profiling was performed. ${ }^{35}$ Apart from these studies, to the best of our knowledge, there are no human-mouse UC comparative studies considering both colon and blood as well as coding and non-coding RNAs to the scale of our study.

Although we recognize that separate analysis of common genes in colon and blood may provide additional information, here, we focus on the genes that were common between both organs in the mouse and human and thus, may represent universal markers of UC. Overall, 51 common PCGs in mouse-human colon and blood with strong inflammatory and immunological profile showed to be consistent. Several of these genes, including ITGAM, STAT3, LCN2, TLR2, PLAUR, JAK3, CD44, OSM, SOCS3, S100A8, S100A9, HP, CFP, IL4R, FES and CSF2RA, have previously been associated to IBD. Interestingly, several less known/studied genes in IBD, including SPI1, FPR2, TYROBP, CKAP4, MCEMP1, ADGRG3, SLC11A1 and SELPLG were identified among top candidates. SPI1 encodes an ETS-domain transcription factor PU.1 that activates gene expression exclusively in hematopoietic cells including both myeloid and lymphoid cells e.g. during lymphocyte B cell development and is involved in disease like Inflammatory diarrhea, primary mediastinal B cell Lymphoma and pediatric $T$ cell acute lymphoblastic leukemia. ${ }^{44,45}$ FPR2 works as a chemoattractant receptor involved in antibacterial host defense and inflammation through sensing of bacteria ${ }^{46}$ which is expressed not only by immune cells, but also epithelial, endothelial and fibroblasts cells ${ }^{47}$ that elicits proinflammatory responses. FPR2 also promotes monocytes inflammatory activities and its absence in knock-out mice results in increased bacteria load in the liver and reduced neutrophil infiltration..$^{48}$ TYROBP encodes a transmembrane signaling polypeptide associated with the killer-cell inhibitory receptor family and plays a role in signal transduction 
and inflammation. ${ }^{49}$ Loss of $T Y R O B P$ has been shown to result in a presenile dementia with bone cysts. ${ }^{50}$ TYROBP expression is increased in Alzheimer's disease (AD) patients and its deficiency in AD mouse showed to be neuroprotective and immune-inflammatory therapeutic which eventually slowed/arrested the progression of pathological late onset sporadic AD. ${ }^{51}$ CKAP4 (also known as CLIMP-63) encodes a transmembrane protein and has been the focus of several investigations (reviewed $\mathrm{in}^{52}$ ) and is believed to regulate cell migration. ${ }^{53}$ MCEMP1 encodes a single-pass transmembrane protein, which is involved in immune responses through mast cell differentiation. ${ }^{54} A D G R G 3$ shows to regulate antimicrobial activity of granulocytes ${ }^{55}$ and seems to be required for macrophages local inflammation development. ${ }^{56}$ SLC11A1, previously known as NRAMP1, is expressed exclusively in immune monocytes and phagocytes like macrophage..$^{57}$ Mutation in these genes may have a role in susceptibility to infections and autoimmune diseases. ${ }^{58}$ SLC11A1 product acidifies the phagosome ${ }^{59}$ and thereby kills the entrapped pathogens. SELPLG's strong association with the immune system has been emphasized before. ${ }^{60}$ This gene codes for PSGL-1 protein, which is a counter receptor for P-selectin that facilitates immune responses by promoting immune effector-cells trafficking into inflamed tissue. These genes are evolutionary conserved and have known interactions with already well-known IBD candidate genes. Thus, these candidates potentially represent new UC diagnostic and therapeutic targets and novel avenues to more detailed disease mechanistic studies.

No long non-coding RNAs made it to the 51 final gene list. The major reason is that only a few IncRNAs are assigned orthology relationships across species in general (e.g. only 131 of all mouse IncRNAs are assigned a human ortholog based on HCOP database). However, we have been able to identify 7 IncRNAs that were SDE in colon in both humans and mice. Apart from $\mathrm{H} 19$ among them, not much is known about the other six IncRNAs in IBD, which clearly shows a need for detailed analysis. We also identified 21 miRNAs SDE in colon in humans and mice. Among them miR-146a-5p, -155-5p, 192-5p, 194-5p, 196b-5p, 200c-3p, 223-3p and 223$5 p$ were frequently shown to be differentially regulated in IBD previously, which makes them promising candidate for further analysis.

Given the complexity of the whole colon and blood cell types, for distinguishing pathogenic mechanisms with higher resolution, one way could be to isolate different cell populations and perform single cell sequencing. One of the limitations of our study is that whole colon and blood gene expression profiling were performed. Albeit, the main rationale was to preserve the natural state of the disease as much as possible. In addition, the disadvantages for isolation of different cell types are the technical fractionation procedures and the time duration from tissue collection to sample processing, which potentially affect gene expression statuses specifically for non-coding RNAs in cells.

\section{Conclusion}

High-throughput transcriptome analysis provides a unique tool to discover new targets for therapeutic and diagnostic applications through identifying relationships between gene expression and disease phenotype. Here, we showed that a one-to-one comparison of the transcriptome of the DSS-UC mouse model to human UC can provide novel disease pathophysiology information by identifying genes and pathways with important contributions in UC. Our data suggest that prospective therapeutic interventions and diagnostic applications should target multiple major gene regulators involved in UC pathogenesis and propagations and combine several genes for valid biomarker applications. Moreover, targeting genes with conserved functional roles in the disease pathogenesis may offer a reliable UC treatment approach compared to targets with different functional roles in different tissues and organisms. 


\section{Conflicts of interest}

TL is employed both by University of Copenhagen and by LEO Pharma A/S. The other authors disclose no conflicts

\section{Author contributions}

R.Y. designed the study, established the mouse model, performed the experiments and interpreted the results and wrote the manuscript. (Conceptualization, Methodology, Software, Validation, Formal analysis, Investigation, Resources, Writing - Original Draft, Visualization, Project administration). O.P. processed and analyzed the RNA-Seq and public datasets and contributed to writing the manuscript (Software, Formal analysis, Data Curation, Writing - Original Draft, Visualization). N.T.D contributed to analyzing the data and to writing the manuscript (Software, Formal analysis, Writing - Original Draft, Visualization). C.A. provided support in analyzing the data and revised the manuscript. (Formal analysis). B.P. and U.H. provided tools and helps for establishing the mouse model. (Methodology, Resources). C.A.S.S performed the qPCRs. (Validation). A.H.M prepared the human samples and revised the manuscript. (Resources). T.L. provided essential insights and revised the manuscript. (Conceptualization). C.H.B.B and M.V prepared the human samples and revised the manuscript. (Resources). J.G. and L.J.J. provided essential tools and insights in designing the experiment, supervised the study and revised the manuscript. (Conceptualization, Supervision, Funding acquisition). F.P. contributed in designing the experiment, provided essential tools and insights, supervised the study and revised the manuscript. (Conceptualization, Supervision, Project administration, Funding acquisition). All authors read, reviewed and approved the final manuscript.

\section{Acknowledgment}

We would like to thank Dr. Charlotte Aaberg Poulsen from University of Southern Denmark for providing the animal study license for UC mouse model establishment. We also would like to thank Professor Poul Flemming Høilund-Carlsen and Christina Baun (Research radiographer) from Clinical Physiology and Nuclear Medicine at Odense University Hospital for performing microPET scanning of the UC mouse models. Moreover, special thanks to Giulia I. Corsi (Ph.D. candidate) from Center for non-coding RNA in Technology and Health for help with the visualization of enrichment analysis.

\section{Funding}

This work has been supported by the Independent Danish Research Foundation, Technology and Production, grants 4005-00443 and 8020-00300B, the Novo Nordisk Foundation, grant NNF14CC0001, Lundbeck foundation, grant R303-2018-3148 and the Sehested Hansen foundation. 


\section{References}

1. Hong SN, Joung JG, Bae JS, et al. RNA-seq Reveals Transcriptomic Differences in Inflamed and Noninflamed Intestinal Mucosa of Crohn's Disease Patients Compared with Normal Mucosa of Healthy Controls. Inflamm Bowel Dis 2017;23:1098-1108.

2. Lekka E, Hall J. Noncoding RNAs in disease. FEBS Lett 2018;592:2884-2900.

3. Casamassimi A, Federico A, Rienzo M, et al. Transcriptome Profiling in Human Diseases: New Advances and Perspectives. Int J Mol Sci 2017;18.

4. Hasler R, Sheibani-Tezerji R, Sinha A, et al. Uncoupling of mucosal gene regulation, mRNA splicing and adherent microbiota signatures in inflammatory bowel disease. Gut 2017;66:2087-2097.

5. Chassaing B, Aitken JD, Malleshappa M, et al. Dextran sulfate sodium (DSS)-induced colitis in mice. Curr Protoc Immunol 2014;104:15 25 1-15 2514.

6. Eichele DD, Kharbanda KK. Dextran sodium sulfate colitis murine model: An indispensable tool for advancing our understanding of inflammatory bowel diseases pathogenesis. World J Gastroenterol 2017;23:6016-6029.

7. Okayasu I, Hatakeyama S, Yamada M, et al. A novel method in the induction of reliable experimental acute and chronic ulcerative colitis in mice. Gastroenterology 1990;98:694-702.

8. Pertea M, Kim D, Pertea GM, et al. Transcript-level expression analysis of RNA-seq experiments with HISAT, StringTie and Ballgown. Nat Protoc 2016;11:1650-67.

9. Friedlander MR, Mackowiak SD, Li N, et al. miRDeep2 accurately identifies known and hundreds of novel microRNA genes in seven animal clades. Nucleic Acids Res 2012;40:37-52.

10. Love MI, Huber W, Anders S. Moderated estimation of fold change and dispersion for RNA-seq data with DESeq2. Genome Biol 2014;15:550.

11. Doncheva NT, Morris JH, Gorodkin J, et al. Cytoscape StringApp: Network Analysis and Visualization of Proteomics Data. J Proteome Res 2019;18:623-632.

12. Shannon P, Markiel A, Ozier O, et al. Cytoscape: a software environment for integrated models of biomolecular interaction networks. Genome Res 2003;13:2498-504.

13. Krämer A, Green J, Pollard J, Jr., et al. Causal analysis approaches in Ingenuity Pathway Analysis. Bioinformatics 2014;30:523-30.

14. Szklarczyk D, Gable AL, Lyon D, et al. STRING v11: protein-protein association networks with increased coverage, supporting functional discovery in genome-wide experimental datasets. Nucleic Acids Res 2019;47:D607-d613.

15. Van der Goten J, Vanhove W, Lemaire K, et al. Integrated miRNA and mRNA expression profiling in inflamed colon of patients with ulcerative colitis. PLoS One 2014;9:e116117.

16. Mo A, Marigorta UM, Arafat $D$, et al. Disease-specific regulation of gene expression in a comparative analysis of juvenile idiopathic arthritis and inflammatory bowel disease. Genome Med 2018;10:48.

17. Ostrowski J, Dabrowska M, Lazowska I, et al. Redefining the Practical Utility of Blood Transcriptome Biomarkers in Inflammatory Bowel Diseases. J Crohns Colitis 2019;13:626-633.

18. Haberman $Y$, Karns R, Dexheimer PJ, et al. Ulcerative colitis mucosal transcriptomes reveal mitochondriopathy and personalized mechanisms underlying disease severity and treatment response. Nat Commun 2019;10:38.

19. Vanhove W, Peeters PM, Staelens D, et al. Strong Upregulation of AIM2 and IFI16 Inflammasomes in the Mucosa of Patients with Active Inflammatory Bowel Disease. Inflamm Bowel Dis 2015;21:267382.

20. Mirza $\mathrm{AH}$, Berthelsen $\mathrm{CH}$, Seemann SE, et al. Transcriptomic landscape of IncRNAs in inflammatory bowel disease. Genome Med 2015;7:39.

21. Lin J, Zhang X, Zhao Z, et al. Novel MicroRNA Signature to Differentiate Ulcerative Colitis from Crohn Disease: A Genome-Wide Study Using Next Generation Sequencing. Microrna 2016;5:222-229.

22. Yates AD, Achuthan P, Akanni W, et al. Ensembl 2020. Nucleic Acids Res 2020;48:D682-D688. 
23. Wright MW, Eyre TA, Lush MJ, et al. HCOP: the HGNC comparison of orthology predictions search tool. Mamm Genome 2005;16:827-8.

24. Seal RL, Gordon SM, Lush MJ, et al. genenames.org: the HGNC resources in 2011. Nucleic Acids Res 2011;39:D514-9.

25. de Lange KM, Moutsianas L, Lee JC, et al. Genome-wide association study implicates immune activation of multiple integrin genes in inflammatory bowel disease. Nat Genet 2017;49:256-261.

26. Jostins L, Ripke S, Weersma RK, et al. Host-microbe interactions have shaped the genetic architecture of inflammatory bowel disease. Nature 2012;491:119-24.

27. Ludwig N, Leidinger $\mathrm{P}$, Becker $\mathrm{K}$, et al. Distribution of miRNA expression across human tissues. Nucleic Acids Res 2016;44:3865-77.

28. Yarani R, Mirza AH, Kaur S, et al. The emerging role of IncRNAs in inflammatory bowel disease. Exp Mol Med 2018;50:1-14.

29. Taman H, Fenton CG, Hensel IV, et al. Transcriptomic Landscape of Treatment-Naïve Ulcerative Colitis. J Crohns Colitis 2018;12:327-336.

30. te Velde AA, de Kort F, Sterrenburg E, et al. Comparative analysis of colonic gene expression of three experimental colitis models mimicking inflammatory bowel disease. Inflamm Bowel Dis 2007;13:32530.

31. Hansen JJ, Holt L, Sartor RB. Gene expression patterns in experimental colitis in IL-10-deficient mice. Inflamm Bowel Dis 2009;15:890-9.

32. Fang $\mathrm{K}$, Bruce $\mathrm{M}$, Pattillo $\mathrm{CB}$, et al. Temporal genomewide expression profiling of DSS colitis reveals novel inflammatory and angiogenesis genes similar to ulcerative colitis. Physiol Genomics 2011;43:43-56.

33. Holgersen $\mathrm{K}$, Kutlu B, Fox $\mathrm{B}$, et al. High-resolution gene expression profiling using RNA sequencing in patients with inflammatory bowel disease and in mouse models of colitis. J Crohns Colitis 2015;9:492506.

34. Rankin CR, Theodorou E, Man Law IK, et al. Identification of novel mRNAs and IncRNAs associated with mouse experimental colitis and human inflammatory bowel disease. Am J Physiol Gastrointest Liver Physiol 2018;315:G722-G733.

35. Czarnewski P, Parigi SM, Sorini C, et al. Conserved transcriptomic profile between mouse and human colitis allows unsupervised patient stratification. Nat Commun 2019;10:2892.

36. Du M, Yuan L, Tan X, et al. The LPS-inducible IncRNA Mirt2 is a negative regulator of inflammation. Nat Commun 2017;8:2049.

37. Chen SW, Wang PY, Liu YC, et al. Effect of Long Noncoding RNA H19 Overexpression on Intestinal Barrier Function and Its Potential Role in the Pathogenesis of Ulcerative Colitis. Inflamm Bowel Dis 2016;22:2582-2592.

38. Li $\mathrm{Y}$, Zhang $\mathrm{S}$, Zhang $\mathrm{C}$, et al. LnCRNA MEG3 inhibits the inflammatory response of ankylosing spondylitis by targeting miR-146a. Mol Cell Biochem 2020;466:17-24.

39. Hu X, Tang J, Hu X, et al. Silencing of Long Non-coding RNA HOTTIP Reduces Inflammation in Rheumatoid Arthritis by Demethylation of SFRP1. Mol Ther Nucleic Acids 2020;19:468-481.

40. Liu R, Tang A, Wang X, et al. Inhibition of IncRNA NEAT1 suppresses the inflammatory response in IBD by modulating the intestinal epithelial barrier and by exosome-mediated polarization of macrophages. Int J Mol Med 2018;42:2903-2913.

41. Neudecker V, Haneklaus M, Jensen $\mathrm{O}$, et al. Myeloid-derived miR-223 regulates intestinal inflammation via repression of the NLRP3 inflammasome. J Exp Med 2017;214:1737-1752.

42. Wang $\mathrm{H}$, Chao K, Ng SC, et al. Pro-inflammatory miR-223 mediates the cross-talk between the IL23 pathway and the intestinal barrier in inflammatory bowel disease. Genome Biol 2016;17:58.

43. Wang H, Zhang S, Yu Q, et al. Circulating MicroRNA223 is a New Biomarker for Inflammatory Bowel Disease. Medicine (Baltimore) 2016;95:e2703.

44. Seki M, Kimura S, Isobe T, et al. Recurrent SPI1 (PU.1) fusions in high-risk pediatric $T$ cell acute lymphoblastic leukemia. Nat Genet 2017;49:1274-1281. 
45. Rothenberg EV, Hosokawa H, Ungerback J. Mechanisms of Action of Hematopoietic Transcription Factor PU.1 in Initiation of T-Cell Development. Front Immunol 2019;10:228.

46. Alessi MC, Cenac N, Si-Tahar M, et al. FPR2: A Novel Promising Target for the Treatment of Influenza. Front Microbiol 2017;8:1719.

47. He HQ, Ye RD. The Formyl Peptide Receptors: Diversity of Ligands and Mechanism for Recognition. Molecules 2017;22.

48. Carp H. Mitochondrial N-formylmethionyl proteins as chemoattractants for neutrophils. J Exp Med 1982;155:264-75.

49. Tomasello E, Vivier E. KARAP/DAP12/TYROBP: three names and a multiplicity of biological functions. Eur J Immunol 2005;35:1670-7.

50. Paloneva J, Kestila M, Wu J, et al. Loss-of-function mutations in TYROBP (DAP12) result in a presenile dementia with bone cysts. Nat Genet 2000;25:357-61.

51. Haure-Mirande JV, Audrain M, Fanutza T, et al. Deficiency of TYROBP, an adapter protein for TREM2 and CR3 receptors, is neuroprotective in a mouse model of early Alzheimer's pathology. Acta Neuropathol 2017;134:769-788.

52. Tuffy KM, Planey SL. Cytoskeleton-Associated Protein 4: Functions Beyond the Endoplasmic Reticulum in Physiology and Disease. ISRN Cell Biology 2012;2012:142313.

53. Osugi Y, Fumoto K, Kikuchi A. CKAP4 Regulates Cell Migration via the Interaction with and Recycling of Integrin. Mol Cell Biol 2019;39.

54. Li K, Wang SW, Li Y, et al. Identification and expression of a new type II transmembrane protein in human mast cells. Genomics 2005;86:68-75.

55. Hsiao CC, Chu TY, Wu CJ, et al. The Adhesion G Protein-Coupled Receptor GPR97/ADGRG3 Is Expressed in Human Granulocytes and Triggers Antimicrobial Effector Functions. Front Immunol 2018;9:2830.

56. Shi J, Zhang X, Wang S, et al. Gpr97 is dispensable for metabolic syndrome but is involved in macrophage inflammation in high-fat diet-induced obesity in mice. Sci Rep 2016;6:24649.

57. Canonne-Hergaux F, Gruenheid S, Govoni G, et al. The Nramp1 protein and its role in resistance to infection and macrophage function. Proc Assoc Am Physicians 1999;111:283-9.

58. Sechi LA, Gazouli M, Sieswerda LE, et al. Relationship between Crohn's disease, infection with Mycobacterium avium subspecies paratuberculosis and SLC11A1 gene polymorphisms in Sardinian patients. World J Gastroenterol 2006;12:7161-4.

59. Lapham AS, Phillips ES, Barton CH. Transcriptional control of Nramp1: a paradigm for the repressive action of c-Myc. Biochem Soc Trans 2004;32:1084-6.

60. Tinoco R, Carrette F, Barraza ML, et al. PSGL-1 Is an Immune Checkpoint Regulator that Promotes T Cell Exhaustion. Immunity 2016;44:1190-203. 
miRNAs, $n=73$

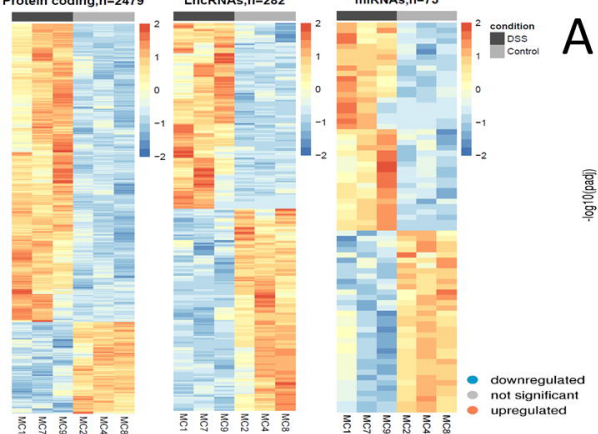

A

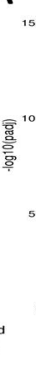

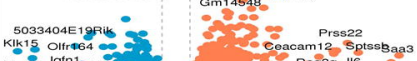

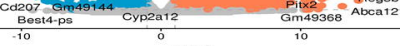
$\log F \mathrm{C}$

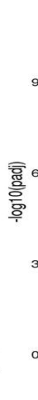

mir-z23-5p

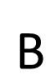

B mir-212-5p

mir-132-3p

mir-96-5p mir-429-3p mir-494-3p mir-182-5p mir-210-3p mir-223-3p mir-192-5p-mir-187-3p -30 mir-132-5p mir-224-5 mir-148a-3p काie 141-3p o mir-615-3p mir-1306-3p mir-213-3p mir-1983

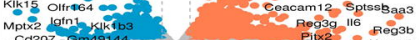

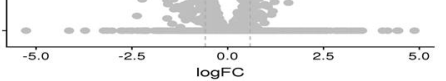

mIRNAs, $n=10$

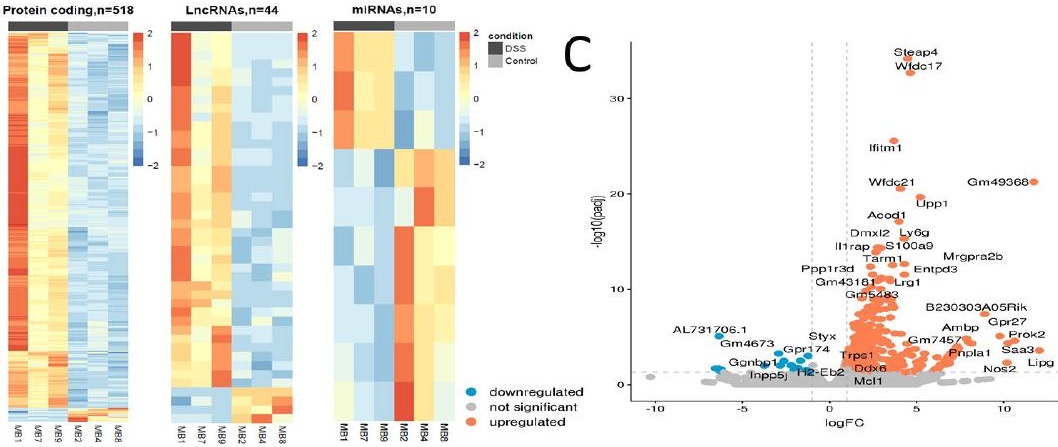

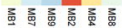

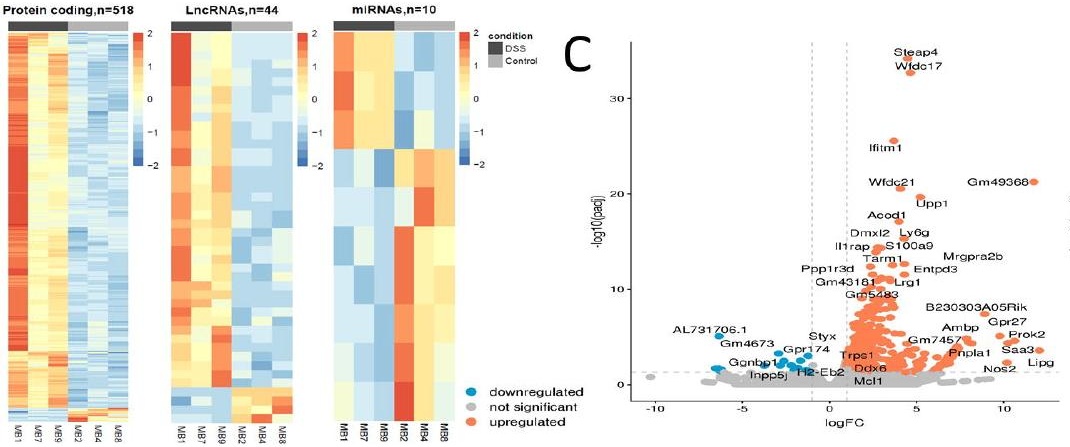

D
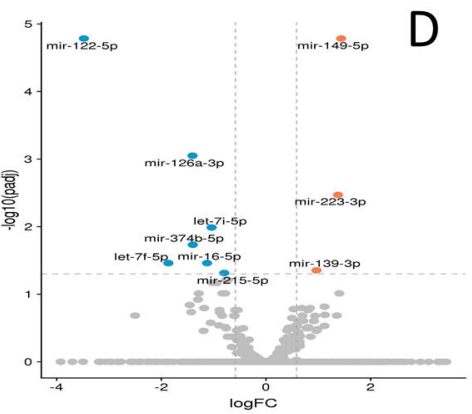
A

\begin{tabular}{lccccc}
\hline Colon & $\begin{array}{c}3782 \\
(84.8 \%)\end{array}$ & $\begin{array}{c}284 \\
(6.4 \%)\end{array}$ & $\begin{array}{c}395 \\
(8.9 \%)\end{array}$ \\
\hline Commonly regulated & Protein coding & IncRNA & miRNA & Others \\
\hline Upregulated & 246 & 5 & 1 & 9 \\
\hline Downregulated & 0 & 0 & 0 & 0 \\
\hline Contra-regulated & 21 & 0 & 0 & 2 \\
\hline
\end{tabular}

C

\section{KEGG pathways}

Staphylococcus aureus infection Osteoclast differentiation Cytokine-cytokine receptor interaction complement and coagulation cascades Hematopoietic cell lineage

$$
\text { Pertussis }
$$

Leishmaniasis

Tuberculosis
Tubmaniasis

NOD-like receptor signaling pathway Jak-STAT signaling pathway Legionellosis Phagosome Neuroactive ligand-receptor interaction Herpes simplex infection

$$
\text { Amoebiasis }
$$

B

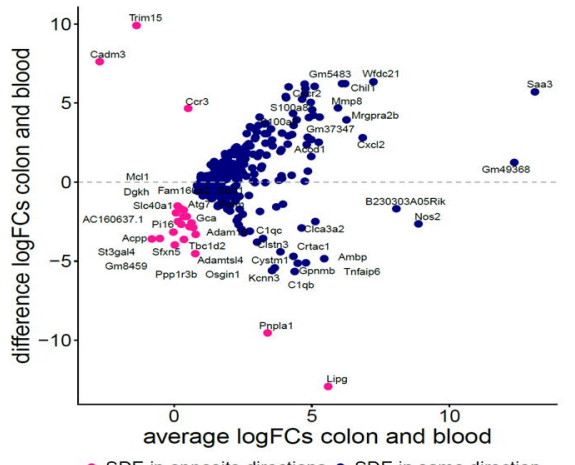

D

\section{GO Biological Processes}

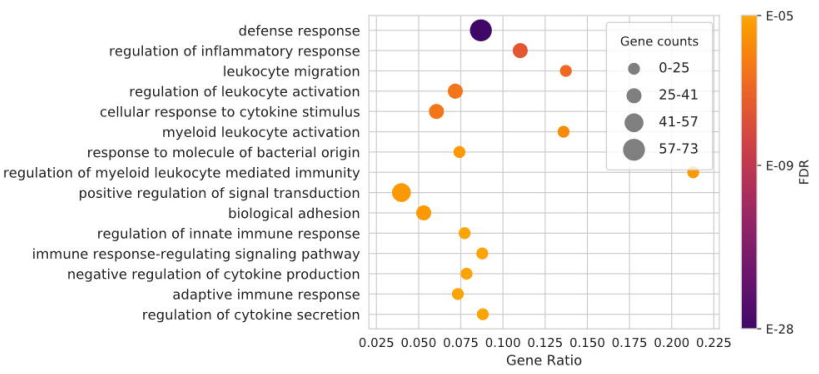


A

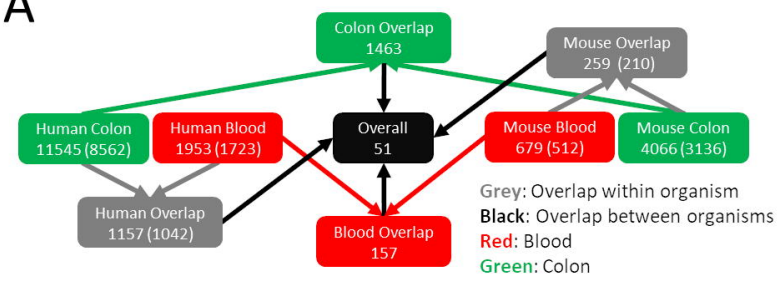

B
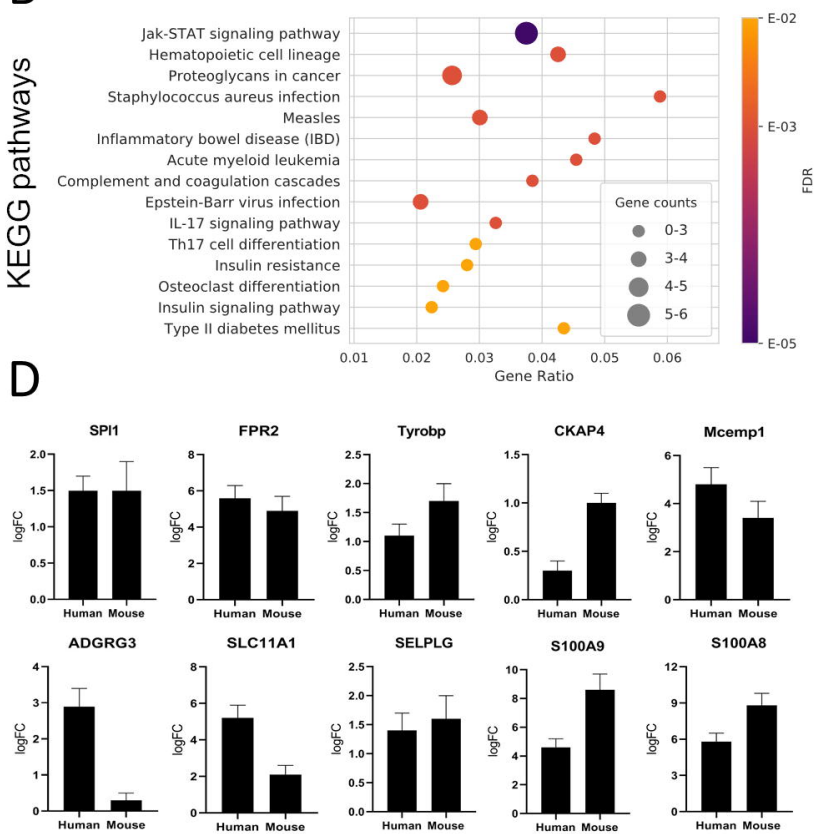

Colon
C
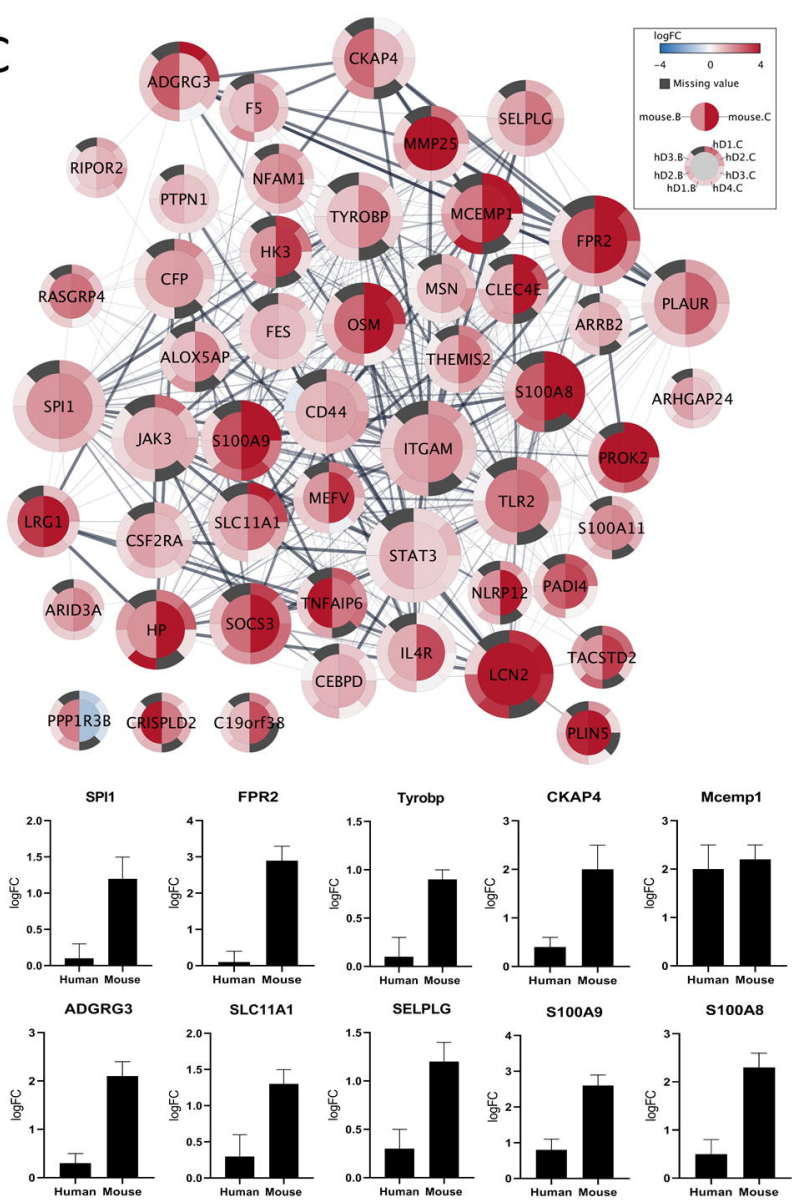


\section{Colon}

\begin{tabular}{|c|c|}
\hline $2479(\Delta 1897, \nabla 582)$ & $518(\boldsymbol{\Delta} 500$ \\
\hline $282\left(\Delta 135, \nabla_{147)}\right.$ & $44\left(\mathbf{\Delta} 40, \nabla_{4)}\right.$ \\
\hline $73\left(\mathbf{\Delta} 39, \nabla_{34}\right)$ & $10(\mathbf{\Delta} 3, \nabla 7)$ \\
\hline $300(\mathbf{\Delta} 201, \mathbf{\nabla 9 9})$ & $54(\mathbf{\Delta} 52, \boldsymbol{\nabla} 2)$ \\
\hline
\end{tabular}

Blood

Upregulation $\boldsymbol{\Delta}$ Downregulation
PCGs

IncRNAs

miRNAs

*Others

*e.g. Pseudogenes, TEC, snoRNA, miscRNA, etc 
GSE109142

GSE117993

Pediatric cohort, treatment-naive

RNA-Seq

Pediatric cohort, treatment-naive

RNA-Seq

GSE59071

Adult cohort

Microarray

Microarray

Adult cohort

GSE67106

Colon Small RNA-Seq

GSE89667

Adult cohort

Small RNA-Se

Illumina Hiseq 2500

GSE48957

Adult cohort

Pediatric cohort

RNA-Seq

Illumina HiSeq 2000, 100 paired-End

RNA-Seq

Ion AmpliSeq Transcriptome

RNA-Seq

Ion AmpliSeq Transcriptome
206 UC, 20 CO

$43 \mathrm{UC}, 55 \mathrm{CO}$

74 UC, 11 CO (colon)

15 UC, 9 CO (colon,or rectum)

10 UC, $18 \mathrm{CO}$ (diverticular disease) 10 UC, $10 \mathrm{CO}$

15 UC, $12 \mathrm{CO}$

34 UC, $35 \mathrm{CO}$

37 UC, 32 CO 
Colon

$\Delta 4658 \nabla 4909 \times 1531$

$\Delta 454 \boldsymbol{\nabla} 673 \times 812$
$\Delta 14 \nabla_{19}$
NA
$\Delta 450 \nabla 181 \times 204$
$\Delta 16 \nabla_{16} \times 6$

*Others 


\section{Colon}

Blood

Human SDE genes with mouse orthologs

8446 (+ 116 miRNAs)

1723

Mouse SDE genes with human orthologs

3066 (+ 70 miRNAs)

512

Common SDE gens in Human and Mouse

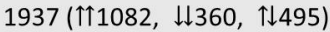

$159(\uparrow \uparrow 154, \downarrow \downarrow 3, \uparrow \downarrow 2)$

Upregulated Downregulated Contra-Regulated Upregulated

Downregulated Contra-Regulated

PCGs

IncRNAs

miRNAs

\begin{abstract}
1061
\end{abstract}
Others (e.g. Pseudogenes, TEC, snoRNA, miscRNA, etc)
5

345
2
12
1

1
489

1

9

7

154
0
NA
0

0

3
0
NA

0
2

0

NA

0 